\title{
Rapid Calculation of Antenna Noise Temperature in Offset Gregorian Reflector Systems
}

\author{
Dirk I. L. de Villiers, Member, IEEE, and Robert Lehmensiek, Senior Member, IEEE
}

\begin{abstract}
Antenna noise temperature calculations of reflector systems is often a slow process and makes direct optimization of the sensitivity of these systems a difficult and time consuming task. This paper presents an improvement to a recently proposed method to speed up these calculations by several orders of magnitude for large dish systems. The accuracy of the improved method is tested for several types of offset Gregorian systems, and errors are shown to be in the order of a few percent. Comparisons of several layers of simplification to a standard brightness temperature model are also presented to aid the designer in the choice of model complexity to use.
\end{abstract}

Index Terms-Noise temperature, Radio astronomy, Reflector antennas.

\section{INTRODUCTION}

Antenna noise temperature is an important metric in the performance of reflector antenna systems used in, for instance, radio astronomy and ground stations [1]. Modern radio telescope instruments like the Square Kilometer Array (SKA) [2] are required to operate over multiple octaves of bandwidth using several feed antennas - all of which have to be optimized for maximum sensitivity. The sensitivity is the ratio of effective aperture area and system noise temperature, which in turn is a combination of antenna and receiver noise. Calculation of the antenna noise temperature requires integration of the product of the radiation pattern of the antenna with the surrounding scene brightness temperature over the entire $4 \pi$ steradian sphere. This so called noise integral is well known and used to calculate the antenna noise temperature, which is simply the radiated power normalized noise integral, as [1]

$$
T_{A}\left(f \mid \hat{\mathbf{r}}_{0}\right)=\frac{\iint_{4 \pi} N\left(f, \theta, \phi \mid \hat{\mathbf{r}}_{0}\right) \sin \theta d \theta d \phi}{\iint_{4 \pi} P(f, \theta, \phi) \sin \theta d \theta d \phi},
$$

where $T_{A}$ denotes the antenna noise temperature and

$$
N\left(f, \theta, \phi \mid \hat{\mathbf{r}}_{0}\right)=T_{b}(f, \theta, \phi) P\left(f, \theta, \phi \mid \hat{\mathbf{r}}_{0}\right),
$$

with $P\left(f, \theta, \phi \mid \hat{\mathbf{r}}_{0}\right)$ the total antenna radiation pattern when pointing in the direction $\hat{\mathbf{r}}_{0}$ at frequency $f$ in the standard spherical coordinate system with azimuthal and zenith angles $\phi$ and $\theta$. The brightness temperature distribution of the scene surrounding the antenna is denoted by $T_{b}(f, \theta, \phi)$. The description for $N$ in (2) is valid for a single medium, and it should, in general, be separated to account for the polarization

This work was financially supported by the National Research Foundation through the South African SKA project.

D.I.L. de Villiers is with the Department of Electrical and Electronic Engineering, Stellenbosch University, Stellenbosch, 7600, South Africa (email: ddv@sun.ac.za)

R. Lehmensiek is with EMSS Antennas (Pty) Ltd. in Technopark, Stellenbosch, 7600, South Africa (e-mail: lehmensk@emss.co.za)

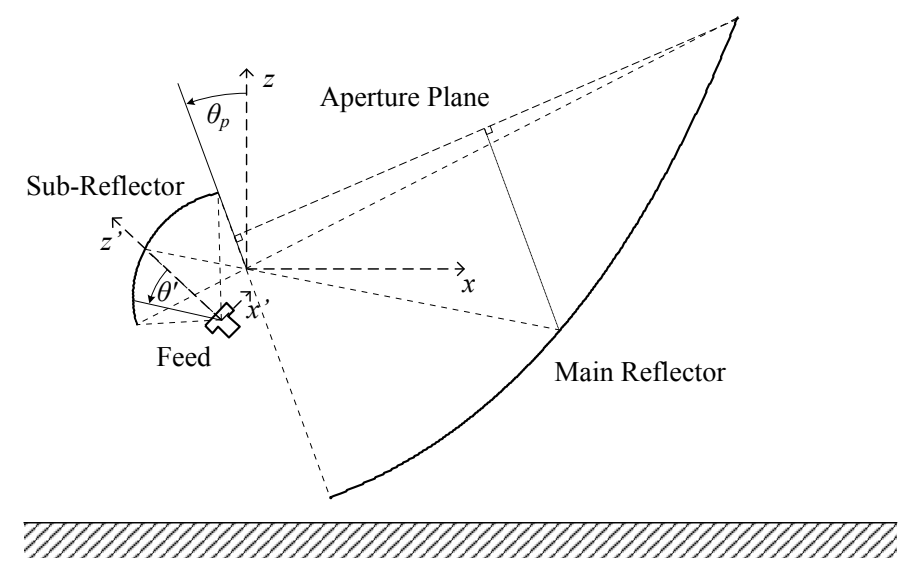

Fig. 1. Symmetry plane cut of a general offset Gregorian reflector system showing the base coordinate system as well as the feed coordinate system (indicated by primes). The reflector is pointing in the $\theta_{p}$ direction, and rotated in the feed-down configuration in this example (indicated by positive $\theta_{p}$ ).

dependent ground emission and scattering. For electrically large antenna systems a prohibitively large number of radiation pattern samples may be required to ensure convergence of the noise temperature integral, and the calculation of the antenna noise temperature, especially when required for a range of tipping angles (the polar angle of the pointing direction of the antenna in a coordinate system with $\mathrm{z}$-axis vertical to the ground), thus becomes slow and a major bottleneck in any sensitivity optimization algorithm.

The SKA will use an offset Gregorian reflector configuration [3], with a symmetry plane cut of a general system shown in Fig. 1. Rapid and accurate evaluation of the antenna noise temperature of the system is imperative to facilitate sensitivity optimization of the feed antennas. First, the brightness temperature of the scene surrounding the antenna must be established. An excellent description of such a model, taking into account most of the physical effects contributing to the brightness temperature, is presented in [4], which will serve as the basis for the brightness temperature used in this paper. However, several simplifications to the model in [4] can be made and have been used in the literature, and descriptions and comparisons of several layers of simplification to the full brightness temperature model in [4] will be presented here. When the brightness temperature has been established, the noise temperature of the antenna system must be calculated. A method to rapidly approximate the antenna noise temperature of offset Gregorian systems has been suggested in [5] and the performance of the approximation evaluated in [6]. It has been shown that the approximation suggested in [5] works well for electrically large reflector systems, but that it typically 
underestimates the noise temperature with increasing error for smaller systems. In this paper an extension to the basic idea of [5] is presented which allows for more accurate approximation of the antenna noise of smaller dishes without significant time penalty. The extended method is also shown to work well for non-standard (conic section) offset Gregorian systems with extended sub-reflectors or shaped reflector surfaces.

\section{Brightness Temperature Model Description}

\section{A. General Formulation}

The brightness temperature models are based on the formulation in [4], with some core equations repeated with a slightly different notation here for clarity.

The basic noise integral is given in (1) and (2), where the role of the brightness temperature distribution is described. Most models for the brightness temperature, including the ones in [4], are rotationally symmetric, and therefore independent of azimuthal variation. In this case different pointing directions of the antenna beam may be accounted for by rotation of the brightness temperature model around a horizontal axis by the angle $\theta_{p}$ (tipping angle), as expanded in [1], [7], and described in terms of the rotated coordinate $\theta^{\prime}\left(\theta_{p}, \theta, \phi\right)$. Equation (2) may now be separated into two regions, the sky contribution and the ground contribution, as

$$
N\left(f, \theta, \phi \mid \hat{\mathbf{r}}_{0}\right)=\left\{\begin{array}{cc}
T_{b}^{s k y}\left(f, \theta^{\prime}\right) P(f, \theta, \phi) & \theta^{\prime} \in[0, \pi / 2) \\
T_{b \|}\left(f, \theta^{\prime}\right) P_{\|}(f, \theta, \phi)+ & \\
T_{b \perp}\left(f, \theta^{\prime}\right) P_{\perp}(f, \theta, \phi) & \theta^{\prime} \in[\pi / 2, \pi] .
\end{array}\right.
$$

The sky brightness temperature contribution is calculated as

$$
\begin{aligned}
T_{b}^{s k y}\left(f, \theta^{\prime}\right)= & T_{b o}(f) e^{-\tau_{f, \theta^{\prime}}\left(0, s_{a}\right)} \\
& +\int_{0}^{s_{a}} \frac{\kappa_{a}\left(f, z^{\prime}\right) T\left(z^{\prime}\right) e^{-\tau_{f, \theta^{\prime}}\left(0, z^{\prime}\right)}}{\sqrt{1-\left(\sin \theta^{\prime} /\left(1+\left(z^{\prime} / r_{e}\right)\right)\right)^{2}}} d z^{\prime},
\end{aligned}
$$

and includes the effects of the absorption by water vapor [8], $\kappa_{\mathrm{H}_{2} \mathrm{O}}(f, z)$, and oxygen [9], $\kappa_{\mathrm{O}_{2}}(f, z)$, as

$$
\kappa_{a}(f, z)=\kappa_{H_{2} O}(f, z)+\kappa_{O_{2}}(f, z) .
$$

The $z$-dependence in (5) is used to implicitly include the altitude variations of the atmospheric pressure and temperature, which are required in the calculations. The background brightness temperature, consisting of the emission from the cosmic microwave background $(\mathrm{CMB})$ and the directional averaged emission from the galaxy, is also included as

$$
T_{b o}(f)=T_{C M B}+T_{g o}\left(f_{0} / f\right)^{\beta},
$$

where $T_{C M B}=2.73 \mathrm{~K}, T_{g o}=20 \mathrm{~K}, f_{0}=408 \mathrm{MHz}$ and $\beta=2.75$ is suggested in [4]. The opacity of the medium, compensated for a curved earth, is calculated as

$$
\tau_{f, \theta^{\prime}}(0, z)=\frac{1}{\sqrt{1-\left(\sin \theta^{\prime} /\left(1+\left(s_{a} / r_{e}\right)\right)^{2}\right.}} \int_{0}^{z} \kappa_{a}(f, \zeta) d \zeta,
$$

where the subscript $\theta^{\prime}$ indicates that the integral should be taken over the path length through the atmosphere at the angle $\theta^{\prime}$. A curved earth model is used in this work with an earth radius of $r_{e}=6370.95 \mathrm{~km}$ and an atmosphere height of $s_{a}=100 \mathrm{~km}$ assumed. The atmospheric temperature profile as a function of altitude, $z$, is denoted by $T(z)$, and any convenient standard atmosphere model may be used to calculate the profile (and similarly for the pressure required in (5)). Interpolants may be extracted for the sky temperature in order to speed up the calculation of (4), as was done in [4].

The ground temperature contribution (due to scattering and emission) is a polarization dependent process, which may be expanded as

$$
\begin{aligned}
T_{b \|}\left(f, \theta^{\prime}\right) & =T_{\|}^{s k y}+T_{\|}^{g n d} \\
& =\Gamma_{\|}\left(\theta_{1}\right) T_{b}^{s k y}\left(f, \theta_{1}\right)+\left[1-\Gamma_{\|}\left(\theta_{1}\right)\right] T_{g n d} \\
T_{b \perp}\left(f, \theta^{\prime}\right) & =T_{\perp}^{s k y}+T_{\perp}^{g n d} \\
& =\Gamma_{\perp}\left(\theta_{1}\right) T_{b}^{s k y}\left(f, \theta_{1}\right)+\left[1-\Gamma_{\perp}\left(\theta_{1}\right)\right] T_{g n d},
\end{aligned}
$$

where $\theta_{1}=\pi-\theta^{\prime}$. The subscripts $\|$ and ${ }_{\perp}$ indicate the parallel and perpendicular polarizations with respect to the plane of incidence at the surface interaction, as shown in [1], [4], with explicit details on the calculation of $P_{\|}$and $P_{\perp}$ from $P$ given in [4]. The ground temperature for all cases in this work is assumed as $T_{\text {gnd }}=300 \mathrm{~K}$. The reflection coefficients are given by

$$
\begin{gathered}
\Gamma_{\|}\left(\theta_{1}\right)=\left|\frac{\cos \theta_{1}-\sqrt{\epsilon_{2}-\sin ^{2} \theta_{1}}}{\cos \theta_{1}+\sqrt{\epsilon_{2}-\sin ^{2} \theta_{1}}}\right|^{2} \\
\Gamma_{\perp}\left(\theta_{1}\right)=\left|\frac{\epsilon_{2} \cos \theta_{1}-\sqrt{\epsilon_{2}-\sin ^{2} \theta_{1}}}{\epsilon_{2} \cos \theta_{1}+\sqrt{\epsilon_{2}-\sin ^{2} \theta_{1}}}\right|^{2},
\end{gathered}
$$

with $\epsilon_{2} \approx 3.5$ for dry land.

\section{B. Brightness Temperature Model Simplifications}

Five brightness temperature models (indicated by integers from 0 to 4 ) of increasing levels of complexity are investigated and compared. All the models assume axial symmetry, and a short description of each is given below.

- Model 4: Ground and sky region temperatures calculated using the full, polarization dependent, model (3), (4) and (8). $T_{A}$ is calculated by using (1).

- Model 3: For unpolarized sources and a high gain antenna not pointing at the ground a polarization averaged reflection coefficient may be defined as

$$
\bar{\Gamma}\left(\theta_{1}\right)=\frac{\Gamma_{\|}\left(\theta_{1}\right)+\Gamma_{\perp}\left(\theta_{1}\right)}{2},
$$

which simplifies (3) to

$$
N(\ldots)=\left\{\begin{aligned}
T_{b}^{s k y} P & \theta^{\prime} \in[0, \pi / 2) \\
{\left[(1-\bar{\Gamma}) T_{g n d}+\bar{\Gamma} T_{b}^{s k y}\right] P } & \theta^{\prime} \in[\pi / 2, \pi],
\end{aligned}\right.
$$

with the frequency and angular dependencies implied to be similar to (3). The sky region temperature is still calculated using (4), and $T_{A}$ is calculated using (1).

- Model 2: Ground region temperature simplified to $270 \mathrm{~K}$ everywhere (see Fig. 2) and the sky region temperature is calculated using (4). $T_{A}$ is calculated using (1). 
- Model 1: Ground region temperature simplified to $270 \mathrm{~K}$ everywhere and the sky region temperature to $0 \mathrm{~K}$ for the calculation of $T_{A}$ using (1). Only half the hemisphere is needed in the integration due to the vanishing sky noise temperature. After integration the sky noise temperature calculated using (4), in the pointing direction, is added to $T_{A}$. This model assumes a high gain antenna where the entire sky noise contribution is due to the main beam.

- Model 0: Ground region temperature simplified to $270 \mathrm{~K}$ everywhere and the sky region temperature to $0 \mathrm{~K}$ for the calculation of $T_{A}$ using (1). Only half the hemisphere is needed in the integration due to the vanishing sky noise temperature. After integration the background brightness temperature, calculated using (6), is added to $T_{A}$. This model assumes a high gain antenna where the entire sky noise contribution is due to the main beam, and a negligible contribution from the atmospheric absorption (typically only valid at frequencies below $10 \mathrm{GHz}$ ). This model has been used for comparative studies during the design phase of the KAT-7 [10] and the Green bank [11] radio telescopes (with the sky temperature assumed $0 \mathrm{~K}$ ).

\section{Comparative Results}

A comparison of the brightness temperature of the different models is shown in Fig. 2.

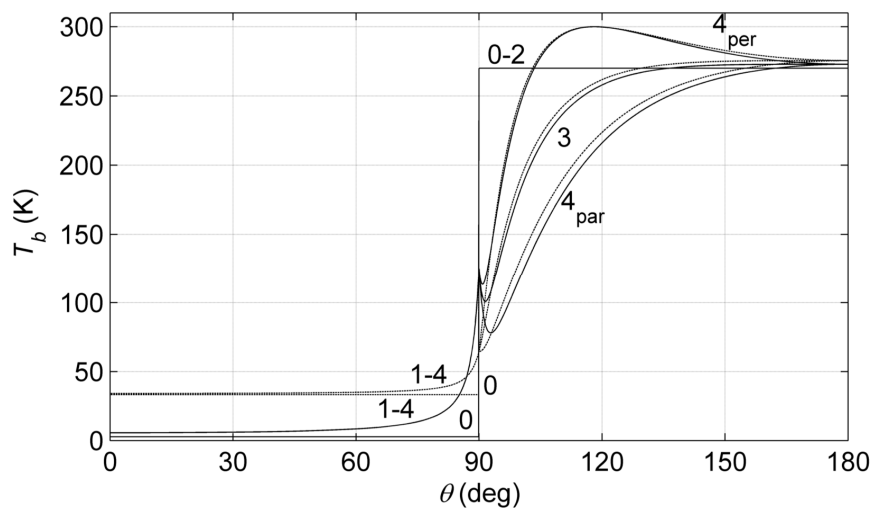

Fig. 2. Comparison of the brightness temperature models at $350 \mathrm{MHz}$ (dashed), and $10 \mathrm{GHz}$ (solid). The model number is shown next to the corresponding trace, as several traces overlap in different regions. The region $\theta<90^{\circ}$ corresponds to the sky, and $\theta \geq 90^{\circ}$ corresponds to the ground.

Note how the simplified Models 0-2 converges to the more complete Models 3-4 towards $0^{\circ}$ and $180^{\circ}$. Also, even though Models 1 and 2 appear identical, they are integrated differently as described in the previous section. The effect of averaging the two polarizations in Model 3 is also clearly visible in the ground region. Finally, the well known increase in sky temperature with decreasing frequency is obvious, while the ground temperature remains virtually frequency independent.

A comparison of the antenna noise temperature produced by using the different models in (1) and (2) is shown in Fig. 3. From Fig. 1 positive tipping angles implies rotation of the dish system with the sub-reflector towards the ground (feed-down), and negative tipping angles with the feed towards the sky (feed-up). For these calculations a simple Gaussian

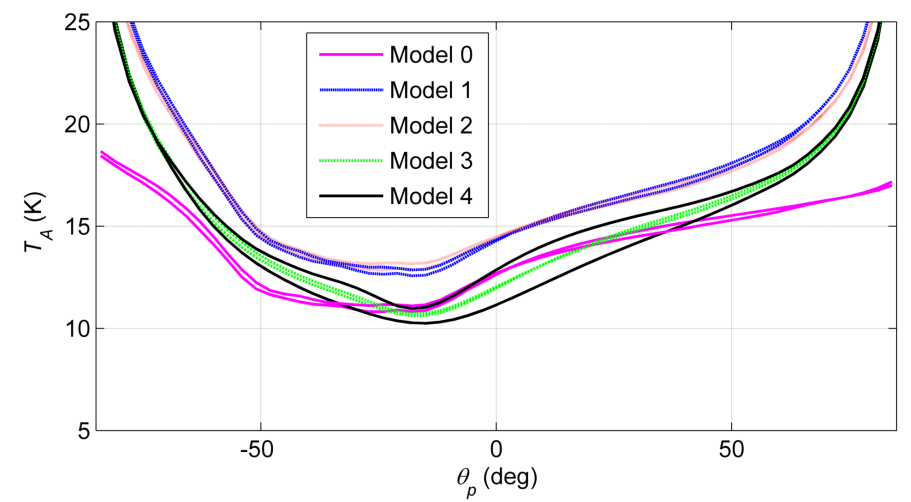

Fig. 3. Comparison of the antenna noise temperature calculated using the different brightness temperature models at $1 \mathrm{GHz}$. Both orthogonal linear polarizations are shown, with the only significant difference seen in Model 4. The bottom trace indicates the polarization in the symmetry plane.

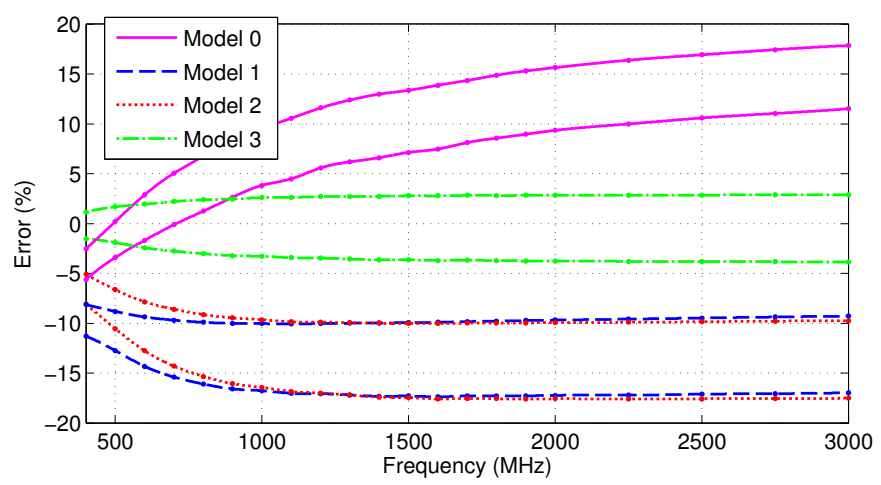

Fig. 4. Tipping angle averaged (over the range $\theta_{p} \in\left[-85^{\circ}, 85^{\circ}\right]$ ) error between the antenna noise temperatures calculated using the different brightness temperature models with Model 4 as base. Markers indicate calculation frequencies. Both orthogonal linear polarizations are shown, with the bottom traces of each type indicating polarizations in the symmetry plane.

feed with an edge taper of $11 \mathrm{~dB}$ was used to illuminate one of the proposed SKA reflector systems described in [3]. All simulations are performed with the commercial code GRASP [12] using physical optics (PO) augmented by physical theory of diffraction (PTD). The specific reflector case used for this illustration has a sub-reflector subtended angle of $\theta_{e}=58^{\circ}$, projected main reflector aperture diameter of $D_{m}=15 \mathrm{~m}$, maximum main and sub-reflector chord lengths of $18.2 \mathrm{~m}$ and $5 \mathrm{~m}$ respectively, and a projected clearance of $0.5 \mathrm{~m}$ between the main and sub-reflectors. To show the performance of the models as a function of frequency, the normalized error using Model 4 as base, averaged over tipping angle, is plotted in Fig. 4. Clearly Model 3 provides the most accurate approximation. Even though the differences between the models may be significant, the simplified models may often be used to find a relative comparison between different dish systems or feeds. For absolute comparisons and noise calculations, the more complete Models 3-4 may be required - depending on the receiver noise temperature. Fig. 4 provides a handy guideline when deciding which noise model to implement for a specific application. Systems with dominant receiver noise can afford less accurate calculation of the antenna noise temperature without significant errors in the sensitivity, and the designer 
may therefore afford to use a simpler noise model in the design process.

\section{MAIN REFLECTOR MASKING}

\section{A. Basic Description}

A recent conference paper has suggested a method to rapidly approximate the antenna noise of offset Gregorian reflector systems [5]. The crux of the method is to remove the main reflector from the calculation domain by assuming all the scattered energy from the sub-reflector (the transmit mode is used for descriptions throughout this work) in the direction of the main reflector is reflected into the main beam and thus toward the sky. The validity of this assumption can be illustrated by comparison of the radiation pattern produced by the full system (including feed, sub-reflector, and main reflector) with that produced by only the feed and sub-reflector combination in Figs. 5 and 6 . Note that the projection

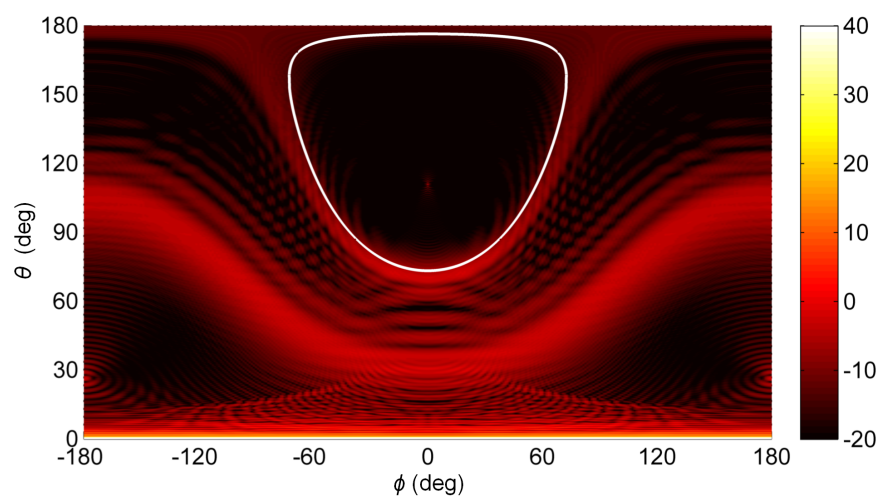

Fig. 5. Radiation pattern (in $\mathrm{dBi}$ ) at $1.5 \mathrm{GHz}$ of the full dual reflector system. The main reflector region is indicated by the region inside the white line.

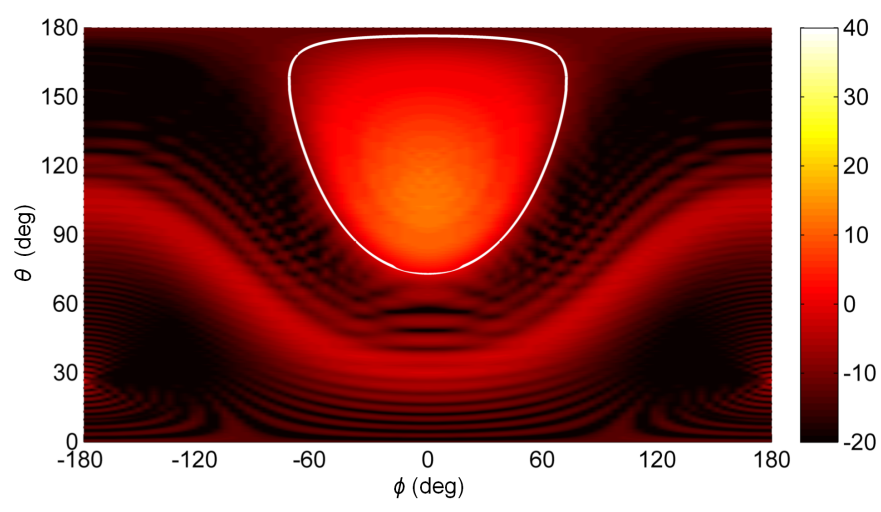

Fig. 6. Radiation pattern (in $\mathrm{dBi}$ ) at $1.5 \mathrm{GHz}$ when only the sub-reflector is included in the simulation. The main reflector region is indicated by the region inside the white line.

used causes similar distortions to the well known Mercator projection used in cartography, where the regions around the poles are stretched. The main beam and first few side lobes in Fig. 5, around $\theta=0^{\circ}$, are stretched to a long horizontal line in $\phi$. Comparing the radiation patterns of the two cases, the main differences are the absence of the main beam around $\theta=0^{\circ}$, and the presence of a large lobe in the direction of the main beam when only the feed and sub-reflector are included in the simulation. Forcing the fields in the main reflector region to zero in Fig. 6 produces a good approximation to the fields in Fig. 5 in the ground region (which, for all tipping angles, has $\theta>15^{\circ}$ in practical applications). The main beam is reconstructed by assuming all the energy in the main reflector region is reflected to the sky, and thus, instead of seeing the ground temperature which is behind the main reflector, actually sees the sky temperature in the pointing direction. It is important to note that a far-field approximation is used here, even though the main reflector is typically in the near field of the sub-reflector. Also, it is assumed that all the energy from the sub-reflector and feed combination is radiated from the origin of the radiation pattern coordinate system, which corresponds to the main reflector primary focus and the subreflector secondary focus.

A plot of the brightness temperature distribution assumed for the main reflector masking method, when pointing at zenith (the distribution is dependent on $\theta_{p}$ ) and using Model 3, is shown in Fig. 7. Calculation of $T_{A}^{0}$ (with the superscript ${ }^{0}$ used to distinguish the approximated temperature from the actual temperature $T_{A}$ ) using (1) is thus accomplished by using this brightness temperature distribution as $T_{b}$, and the radiation pattern depicted in Fig. 6 as $P$.

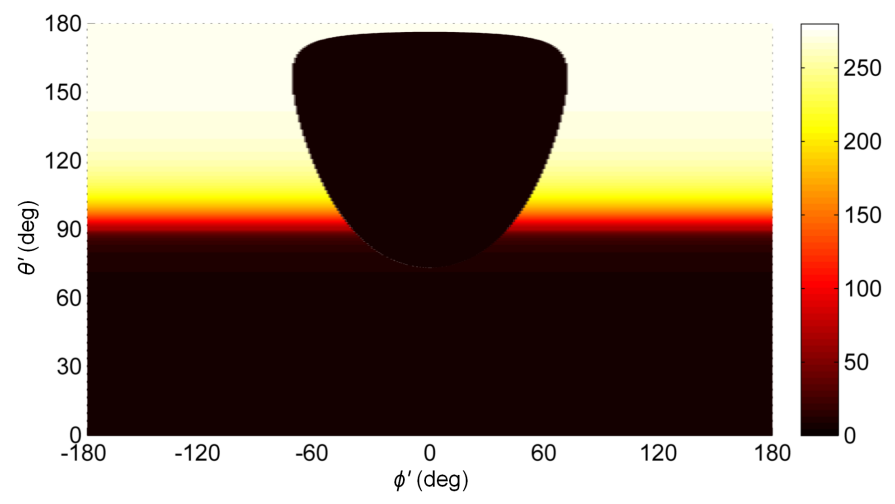

Fig. 7. Model 3 brightness temperature distribution in $\mathrm{K}$, for zenith pointing, used in the main reflector masking approximation.

The integration grid required to ensure a converged noise integral, (1), becomes finer with increasing antenna system size, with a rule of thumb for the sample spacing given in [6]. Calculation of the radiation pattern and noise temperature of the sub-reflector and feed combination is faster than for the full system because only one reflector is required and fewer pattern samples are needed for convergence of the noise integral. This main reflector masking method has proved accurate for large reflectors, with significant speed up over the standard method achieved [5], [6].

When the electrical size of the system is reduced, the accuracy of the main reflector masking method is also reduced. This is due to edge diffraction effects from the main reflector which are ignored in the basic geometric optics masking procedure described above and in [5]. Edge diffraction causes energy scattered from the sub-reflector to illuminate the shadow region of the main reflector which is typically pointed towards ground. Assuming all the energy from the sub-reflector in the direction of the main reflector is reflected into the main beam 


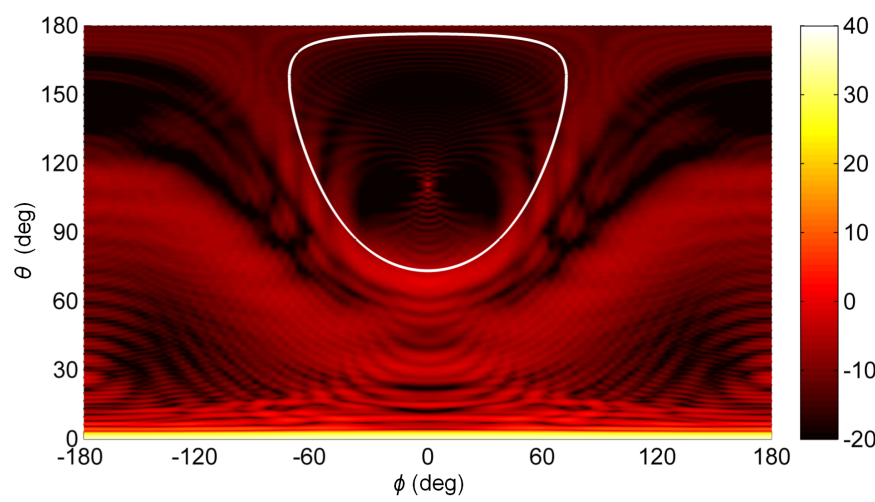

Fig. 8. Radiation pattern (in $\mathrm{dBi}$ ) at $500 \mathrm{MHz}$ of the full dual reflector system. The main reflector region is inside the white line.

will thus typically underestimate the noise temperature, since some of this energy is not reflected towards the cold sky, but diffracted towards the hot ground. This effect is illustrated in Fig. 8, which is the same simulation as Fig. 5, but performed at $500 \mathrm{MHz}$. Note the increased energy density in the region behind the main reflector.

The rest of this section describes a simple method to compensate for this diffraction effect and improve the lowfrequency accuracy of the main reflector mask noise temperature approximation without significantly increasing the calculation time.

\section{B. Diffraction Compensation}

When using the main reflector masking method to approximate the antenna noise temperature, it is assumed all the energy in the direction of the main reflector sees the sky temperature in the pointing direction. Diffraction of energy behind the main reflector, however, causes an effective increase of background noise temperature for the energy in the direction of the main reflector mask. Since the fine structure of the diffracted energy is not available from simulations where the main reflector is omitted, this increased temperature will be compensated for in an average sense. Defining a correction factor, $\alpha\left(f, \theta_{p}\right) \in[0,1]$, the effective brightness temperature, $T^{\alpha}\left(f, \theta_{p}\right)$, for all the energy propagating towards the main reflector mask may be formulated as

$$
\begin{aligned}
& T^{\alpha}\left(f, \theta_{p}\right)=\left[1-\alpha\left(f, \theta_{p}\right)\right] T^{r}+\alpha\left(f, \theta_{p}\right) \overline{T^{d}} \\
& T_{\|}^{\alpha}\left(f, \theta_{p}\right)=\left[1-\alpha\left(f, \theta_{p}\right)\right] T^{r}+\alpha\left(f, \theta_{p}\right) T_{\|}^{d} \\
& T_{\perp}^{\alpha}\left(f, \theta_{p}\right)=\left[1-\alpha\left(f, \theta_{p}\right)\right] T^{r}+\alpha\left(f, \theta_{p}\right) T_{\perp}^{d}
\end{aligned}
$$

with

$$
\overline{T^{d}}=\frac{T_{\|}^{d}+T_{\perp}^{d}}{2} .
$$

$T^{d}$ indicates the approximate brightness temperature behind the reflector,

$$
\begin{aligned}
& T_{\|}^{d}\left(f, \theta_{p}\right)=T_{b \|}\left(f, \theta_{d}\right) \\
& T_{\perp}^{d}\left(f, \theta_{p}\right)=T_{b \perp}\left(f, \theta_{d}\right),
\end{aligned}
$$

with $\theta_{p}$ the tipping angle and $\theta_{d}=\theta_{p}+\pi$ (wrapped), and $T^{r}$ the brightness temperature in the direction of the main beam

$$
T^{r}\left(f, \theta_{p}\right)=T_{b}^{s k y}\left(f, \theta_{p}\right) \text {. }
$$

Equation (2) may be expanded into the ground and sky regions explicitly as

$$
N=N_{G N D}+N_{S K Y}
$$

where the frequency and angular dependence is implied, with

$$
N_{G N D}=P_{\|}^{g}\left(M T_{\|}^{\alpha}+W T_{b \|}\right)+P_{\perp}^{g}\left(M T_{\perp}^{\alpha}+W T_{b \perp}\right),
$$
and

$$
P_{x}^{g}(f, \theta, \phi)=\left\{\begin{aligned}
P_{x}(f, \theta, \phi) & \text { ground region } \\
0 & \text { sky region. }
\end{aligned}\right.
$$

The subscript ${ }_{x}$ indicates either $\|$ or $\perp$ implying the radiation pattern power in the ground region in the parallel and perpendicular polarizations (with respect to the surface interaction) respectively. Only the feed and sub-reflector combinations are used to calculate all radiation patterns in this section, unless specifically stated otherwise. The functions $M$ and $W$ are used to indicate the masked and unmasked regions respectively, where

$$
M(\theta, \phi)= \begin{cases}1 & \text { inside main reflector mask } \\ 0 & \text { outside main reflector mask, }\end{cases}
$$

and $W(\theta, \phi)=1-M(\theta, \phi)$.

For the sky region,

$$
N_{S K Y}=P^{s}\left(M T^{\alpha}+W T_{b}^{s k y}\right)
$$

where the frequency and angular dependence is implied, and

$$
P^{s}(f, \theta, \phi)=\left\{\begin{aligned}
0 & \text { ground region } \\
P(f, \theta, \phi) & \text { sky region. }
\end{aligned}\right.
$$

The total power integral in the denominator in (1) is denoted as

$$
I_{t}(f)=\iint_{4 \pi} P(f, \theta, \phi) \sin \theta d \theta d \phi .
$$

By substituting (12) into (17) and (20), and expanding (16) into (1), $\alpha$ may be solved as

$$
\alpha\left(f, \theta_{p}\right)=\frac{T_{A} I_{t}-I_{w}-I_{r}}{I_{d}-I_{r}},
$$

with (integrand frequency and angular dependence implied)

$$
\begin{aligned}
I_{r}\left(f, \theta_{p}\right) & =\iint_{4 \pi} M\left(P^{s}+P_{\|}^{g}+P_{\perp}^{g}\right) T^{r} \sin \theta d \theta d \phi \\
I_{d}\left(f, \theta_{p}\right) & =\iint_{4 \pi} M\left(P^{s} \overline{T^{d}}+P_{\|}^{g} T_{\|}^{d}+P_{\perp}^{g} T_{\perp}^{d}\right) \sin \theta d \theta d \phi \\
I_{w}\left(f, \theta_{p}\right) & =\iint_{4 \pi} W\left(P^{s} T_{b}^{s k y}+P_{\|}^{g} T_{\|}+P_{\perp}^{g} T_{\perp}\right) \sin \theta d \theta d \phi .
\end{aligned}
$$

For the polarization averaged models ( 0 to 3 ), equations (16) to (21) simplify to

$$
N=P\left[M T^{\alpha}+W T_{b}\right]
$$

and (24) reduces to

$$
\begin{aligned}
I_{r}\left(f, \theta_{p}\right) & =\iint_{4 \pi} M P T^{r} \sin \theta d \theta d \phi \\
I_{d}\left(f, \theta_{p}\right) & =\iint_{4 \pi} M P \overline{T^{d}} \sin \theta d \theta d \phi \\
I_{w}\left(f, \theta_{p}\right) & =\iint_{4 \pi} W P T_{b} \sin \theta d \theta d \phi .
\end{aligned}
$$


Solution of $\alpha$ in (23) is, of course, dependent on the availability of a solution for the antenna noise temperature, $T_{A}$. An approximate solution, $\alpha^{\prime}\left(f, \theta_{p}\right) \approx \alpha\left(f, \theta_{p}\right)$ may be constructed as

$$
\alpha^{\prime}\left(f, \theta_{p}\right)=\alpha\left(f_{1}, \theta_{p}\right) \frac{f_{1}}{f} .
$$

The inverse frequency dependence is deduced from the diffraction coefficients in the geometric theory of diffraction, since the magnitude of $\alpha$ is proportional to the diffracted energy behind the main reflector. An approximate correction factor $\alpha^{\prime}$ can thus be calculated by using (23) at a single frequency $f_{1}$, where $T_{A}$ is calculated using the full reflector system including the main reflector, and expanded to a smooth function in frequency using (27). The frequency $f_{1}$ is normally chosen as the lowest frequency of interest to minimize the time required for the antenna noise calculation. Once $\alpha^{\prime}$ is known it is used in (12) to calculate the effective brightness temperature for the masked region $M$, which in turn is used in (16), (17), and (20), or (25), to calculate the approximated antenna noise temperature $T_{A}^{\prime}$ using (1).

\section{RESUlts}

To evaluate the performance of the approximation method presented in Section III, different feed types were used to illuminate several offset Gregorian type dishes, and the accuracy of the main reflector masking methods were evaluated. At each frequency point the full dual reflector system radiation pattern was simulated and used to calculate the reference antenna noise temperature $T_{A}$ as well as the correction factor $\alpha$ for a range of tipping angles $\theta_{p} \in\left[-85^{\circ}, 85^{\circ}\right]$. Additionally, the main reflector masking method was used to calculate $T_{A}^{0}$ and $T_{A}^{\prime}$ corresponding to no compensation and compensation using $\alpha^{\prime}$ respectively. GRASP PO and PTD simulations were used in all cases. All 5 the suggested brightness temperature models of Section II were evaluated, and similar performance was achieved for all cases. To conserve space, results shown will be limited to those obtained by using Model 3 .

\section{A. Ideal Feed Example}

First, an ideal feed with assumed Gaussian radiation pattern is used to illuminate the dish system described in Section II-C. An $11 \mathrm{~dB}$ edge taper was used, and the frequency range evaluated is $f \in[0.4 \mathrm{GHz}, 3.0 \mathrm{GHz}]$. A comparison of the correction factors, averaged over tipping angles, is shown in Fig. 9. The $1 / f$ frequency response used in $\alpha^{\prime}$ is seen to be a good approximation to $\alpha$. Note here again that only the first frequency point of $\alpha$ is used to calculate $\alpha^{\prime}$.

The percentage errors, normalized to $T_{A}$, is shown for $T_{A}^{0}$ and $T_{A}^{\prime}$ over frequency and tipping angle in Fig. 10. The maximum and mean errors for the respective approximations and compensations are given in Table I, where both orthogonal linear polarizations are considered over the full frequency and tipping domains. A significant improvement in accuracy of the masking approximation is observed when the diffraction compensation is used. Similar results were found for different edge taper values and dish geometries. The speed-up factor for this system when using the compensated approximation is in the order of 130 .

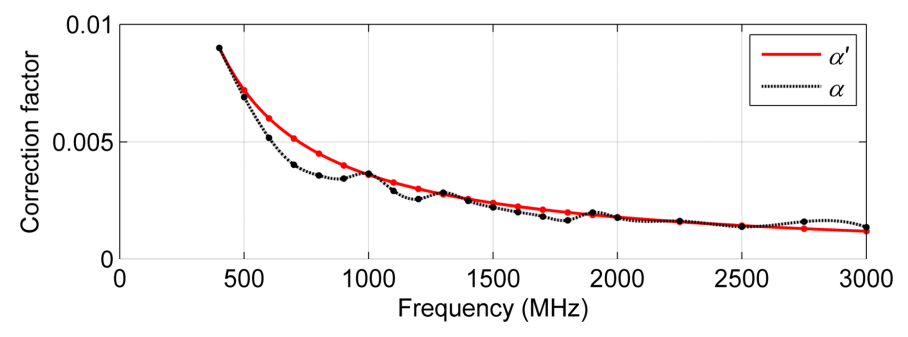

Fig. 9. Correction factors for an ideal Gaussian feed with $11 \mathrm{~dB}$ edge taper averaged over tipping angle. The feed is linearly polarized orthogonal to the plane of symmetry.

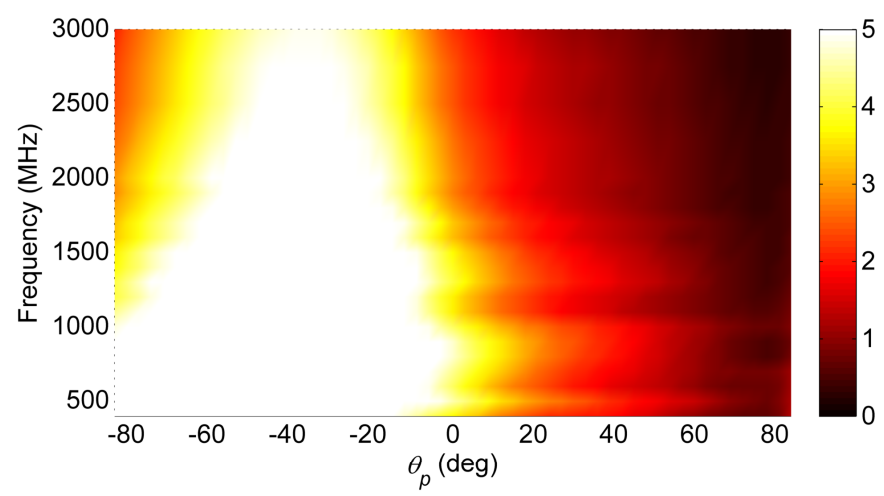

(a)

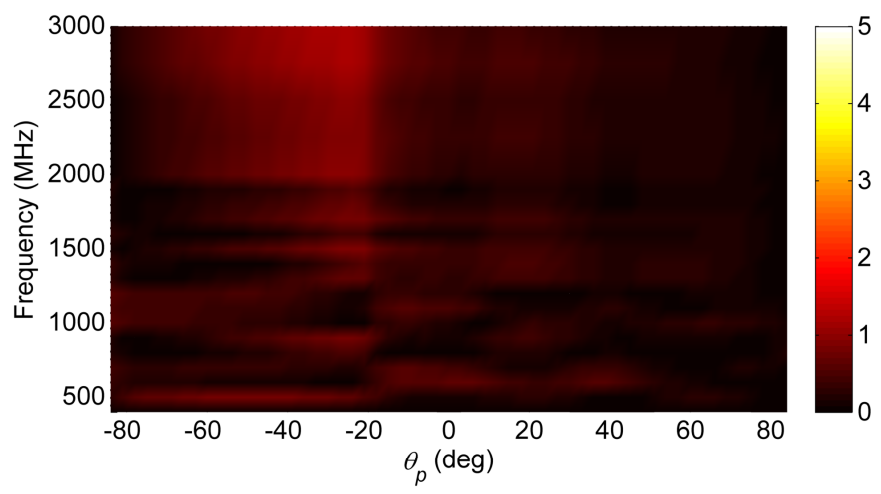

(b)

Fig. 10. Percentage errors in noise temperature when calculated using the main reflector masking approximations for an ideal Gaussian feed with $11 \mathrm{~dB}$ edge taper. Errors for $T_{A}^{0}$ is shown in (a) and $T_{A}^{\prime}$ in (b). The feed is linearly polarized orthogonal to the plane of symmetry.

\section{B. Horn Feed Example}

The performance of the approximation was also evaluated, on the same reflector system as before, when using corrugated horn feeds of the type described in [13]. Three horns are analyzed, with names and operating bands given by: Horn1 - [350 MHz, $640 \mathrm{MHz}$ ], Horn2 - [580 MHz, $1010 \mathrm{MHz}$, and Horn3 - [950 MHz, $1760 \mathrm{MHz}$. Here the feed patterns have some variation with frequency, with the edge illumination level of Horn1 varying between $-18 \mathrm{~dB}$ and $-8 \mathrm{~dB}$, and that of Horn2 and Horn3 varying between about $-15.5 \mathrm{~dB}$ and $-12.5 \mathrm{~dB}$. The back lobe radiation is relatively low for all three horns, with the maximum front-to-back ratio of Horn 1 about $-24 \mathrm{~dB}$, Horn 2 about $-26 \mathrm{~dB}$, and Horn3 about $-29 \mathrm{~dB}$. From Fig. 11 and Table $\mathrm{I}$ it is clear that the diffraction compensation improves the approximation significantly. 
Again, in Table I both polarizations and the full tipping and frequency domains are considered. The speed-up factor when using the compensated approximation in the high band is in the order of 90 .

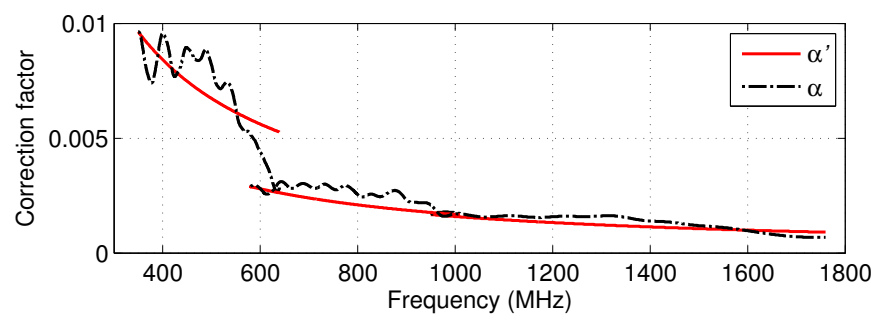

Fig. 11. Correction factors for three overlapping corrugated horn feeds averaged over tipping angle. The feeds are linearly polarized orthogonal to the plane of symmetry. The discontinuities around $600 \mathrm{MHz}$ and $1000 \mathrm{MHz}$ are due to the use of different horns on the same plot.

TABLE I

ERROR COMPARISON FOR MAIN REFLECTOR MASKING WITH AND WITHOUT COMPENSATION

\begin{tabular}{lllccc}
\hline \multirow{2}{*}{ Feed } & \multirow{2}{*}{ Band (MHz) } & \multicolumn{3}{c}{ Mean Error (\%) } & \multicolumn{2}{c}{ Max Error (\%) } \\
& & $T_{A}^{0}$ & $T_{A}^{\prime}$ & $T_{A}^{0}$ & $T_{A}^{\prime}$ \\
\hline \hline Gaussian & $500-3000$ & 3.6 & 0.4 & 8.7 & 2.2 \\
Horn1 & $350-640$ & 4.8 & 1.2 & 10.7 & 6.1 \\
Horn2 & $580-1010$ & 3.6 & 0.6 & 7.7 & 2.5 \\
Horn3 & $950-1760$ & 3.0 & 0.5 & 6.5 & 2.1 \\
QRFH & $500-2000$ & 5.7 & 2.9 & 12.0 & 8.0 \\
\hline
\end{tabular}

\section{Wide Band Feed Example}

When a feed with significant back lobe radiation is used, the masking approximation is expected to perform poorly. This is due to the assumption that the sub-reflector and feed combination radiation mainly emanates from the secondary focus of the sub-reflector which becomes invalid. Back lobe radiation from the feed will not be handled correctly in the masking approximation, since the main reflector mask is defined in terms of the secondary sub-reflector focus, which is not where the feed is placed. Considering Fig. 1, radiation from the feed in the $\theta_{p}+\pi$ direction will actually miss the main reflector, where the main reflector mask includes this direction for the illustrated case. The examples considered in the previous sections had very low back lobe radiation levels with negligible effect on the results.

As an example of a system with significant back lobe radiation a quad-ridged flared horn $(\mathrm{QRFH})$, similar to the type discussed in [14], is used to evaluate the performance of the approximations over a 4:1 bandwidth. The specific feed has a maximum front-to-back ratio of around $-12 \mathrm{~dB}$, with a main beam showing significant frequency variation with edge illumination levels varying between $-8 \mathrm{~dB}$ and $-19 \mathrm{~dB}$. A comparison of the correction factors is shown in Fig. 12. Note how the reference correction factor, $\alpha$, deviates significantly from the expected $1 / f$ response, since more of the back lobe radiation is masked than should actually be

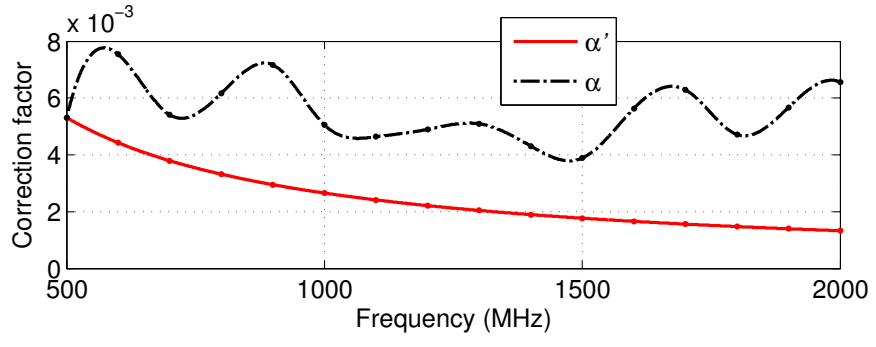

Fig. 12. Correction factors for a QRFH averaged over tipping angle. The feed is linearly polarized orthogonal to the plane of symmetry.

the case, causing underestimated masked noise temperature approximations. The mean and maximum errors, over the full range of tipping angles and frequencies, are shown in Table I. Even in this extreme case the compensation still improves the approximation by about $50 \%$ reduction in average noise temperature error.

\section{Performance on Several Dish Systems}

To evaluate the performance of the approximations on different reflector systems, Horn3 used in Section IV-B was used to illuminate a wide variety of offset Gregorian reflector systems. In total 18 different unshaped systems, all described in [3], were evaluated over the full feed horn operating range. In addition to the unshaped systems, five shaped offset Gregorian type systems were also evaluated using the same feed horn. The shaping algorithm used is described in [15], and the specific mapping chosen to maximize the sensitivity of the system when a $12 \mathrm{~dB}$ edge taper Gaussian feed is used, while maintaining second side lobe levels below $-30 \mathrm{~dB}$. The unshaped results are summarized in the rows marked "U", and the shaped results in the rows marked "S", in Table II. The

TABLE II

ERROR COMPARISON FOR MAIN REFLECTOR MASKING WITH AND WITHOUT COMPENSATION USING A CORRUGATED HORN FEED

\begin{tabular}{ccccccccccccc}
\hline \multicolumn{1}{c}{ Mean Error (\%) } & \multicolumn{5}{c}{ Max Error (\%) } \\
\multicolumn{1}{c}{$\theta_{p}:$} & $-70^{\circ}$ & $-35^{\circ}$ & $0^{\circ}$ & $35^{\circ}$ & $70^{\circ}$ & $-70^{\circ}$ & $-35^{\circ}$ & $0^{\circ}$ & $35^{\circ}$ & $70^{\circ}$ \\
\hline \hline \multirow{2}{*}{$\mathrm{U} T_{A}^{0}$} & 5.3 & 7.0 & 4.8 & 2.9 & 1.8 & 7.7 & 10.4 & 9.2 & 4.5 & 3.0 \\
& $T_{A}^{\prime}$ & 0.9 & 1.3 & 0.9 & 0.6 & 0.5 & 2.7 & 4.2 & 3.8 & 1.8 & 1.3 \\
\hline \multirow{2}{*}{$\mathrm{S}$} & $T_{A}^{0}$ & 10.5 & 13.8 & 6.2 & 3.0 & 1.5 & 12.7 & 17.0 & 9.8 & 5.6 & 2.5 \\
& $T_{A}^{\prime}$ & 1.9 & 2.7 & 1.2 & 0.8 & 0.5 & 3.8 & 5.6 & 3.2 & 1.8 & 1.0 \\
\hline
\end{tabular}

improvement of the compensated approximations is again clear and, importantly, the results for the shaped systems show that the compensated approximations are still valid - albeit with a slightly larger error. This result is significant, since for shaped systems radiation from the origin is no longer focused.

\section{E. Discussion}

The parameter of interest when calculating antenna noise is actually the sensitivity of the system, not the noise temperature or the correction factor. For the frequencies of interest here (below $10 \mathrm{GHz}$ ), the antenna noise temperature is typically reduced with an increase in frequency, and large errors at high 
frequency may actually imply relatively small absolute errors. Large errors on small antenna noise numbers may still lead to accurate sensitivity results when the system becomes receiver noise dominated, as is typically the case at higher frequencies.

Large back lobe radiation from the feed causes the correction to become less accurate and typically underestimate the antenna noise. However, the correction still provides an improved estimate over the simple masking method, since at least the main reflector diffraction is accounted for.

Finally, the general main reflector masking method is more accurate when rotating the reflector system feed down than when rotating feed up. This is due to the fact that the majority of the noise temperature contribution for the feed down case is due to the sub-reflector diffraction cone, or feed energy spilling past the sub-reflector, as described in [16]. For feed down rotation, an increasingly smaller percentage of the antenna noise contribution is due to the energy spilling past the main reflector as the tipping angle is increased. Therefore errors in the calculation of this contribution become less important, as is seen through all the results presented where the masking approximations become increasingly more accurate as the tipping angle is increased in the feed down configuration.

\section{CONClusion}

An improved method for the rapid calculation of antenna noise temperature in offset Gregorian reflector systems was presented. The method relies on a previously described masking method, where the main reflector is removed from the calculation domain, and the sky noise is projected onto the region where the main reflector would have been. This method typically under-estimates the noise since diffraction behind the main reflector is ignored. A compensation method was suggested to account for the diffracted energy behind the main reflector, which only requires an additional analysis of the full system at a single frequency. Several reflector systems and feeds were simulated and results show that errors in the order of a few percent can be expected for most highly directional feeds - a significant improvement over the non-compensated masking approximation. A set of brightness temperature models and simplifications was also presented and compared.

\section{REFERENCES}

[1] T. Y. Otoshi, Noise Temperature Theory and Applications for Deep Space Communications Antenna Systems. Artech House, 2008.

[2] P. E. Dewdney, P. J. Hall, R. T. Schilizzi, and T. J. L. W. Lazio, "The Square Kilometer Array," Proceedings of the IEEE, vol. 97, no. 8, pp. 1482 - 1496, Aug. 2009.

[3] I. P. Theron, R. Lehmensiek, and D. I. L. de Villiers, "Towards an optics design for SKA," in IEEE AFRICON, Mauritius, Sep. 2013.

[4] G. C. Medellin, "Antenna noise temperature calculations," in SKA Memo 95, Jul. 2007

[5] W. A. Imbriale, "Faster antenna noise temperature calculations using a novel approximation technique," in Antennas and Propagation Society International Symposium (APSURSI), 2010 IEEE, Toronto, ON, Canada, Jul. 2010 .

[6] D. I. L. de Villiers and R. Lehmensiek, "Efficient simulation of radiometric noise in offset Gregorian antenna systems," in Proc. 7th Eur. Conf. Antennas and Propag. (EuCAP), Gothenburg, Sweden, Apr. 2013, pp. $3357-3359$.
[7] J. Dijk, H. H. H. Groothuis, and E. J. Maanders, "Some improvements in antenna noise temperature calculation," IEEE Trans. Antennas Propag., vol. 18 , no. 5, pp. 690-692, Sep. 1970.

[8] F. T. Ulaby, R. K. Moore, and A. K. Fung, Microwave Remote Sensing. Addison-Wesley Publishing Company, 1981, vol. 1, ch. 4-5.

[9] P. W. Rosenkranz, "Shape of the 5mm oxygen band in the atmosphere," IEEE Trans. Antennas Propag., vol. AP-23, pp. 498-506, 1975.

[10] R. Lehmensiek and I. P. Theron, "L-band feed horn and orthogonal mode transducer for the KAT-7 radio telescope," IEEE Trans. Antennas Prapag., vol. 59, no. 6, pp. 1894-1901, Jun. 2011.

[11] S. Srikanth, "Comparison of spillover loss of offset Gregorian and cassegrain antennas," in Antennas and Propagation Society International Symposium, AP-S. Digest, vol. 1, Jun. 1991, pp. 444-447.

[12] TICRA, Copenhagen, Denmark. GRASP10, Version 10.0.1. [Online]. Available: http://www.ticra.com

[13] R. Lehmensiek and D. I. L. De Villiers, "Wide flare angle axially corrugated conical horn design for a classical offset dual-reflector antenna," in 6th European Conference on Antennas and Propagation (EUCAP), Prague, Czech Republic, Mar. 2012, pp. 3292-3294.

[14] T. S. Beukman, M. Ivashina, R. Maaskant, P. Meyer, and C. Bencivenni, "A quadraxial feed for ultra-wide bandwidth quadruple-ridged flared horn antennas," in The 8th European Conference on Antennas and Propagation (EuCAP), The Hague, Netherlands, Apr. 2014.

[15] P.-S. Kildal, "Synthesis of multireflector antennas by kinematic and dynamic ray tracing," IEEE Trans. Antennas Propag., vol. 38, no. 10, pp. 1587-1599, Oct. 1990.

[16] D. I. L. De Villiers and R. Lehmensiek, "Offset Gregorian reflector shaping for optimum sensitivity," in Proc. Int. Conf. Electromagn. Adv. Appl. (ICEAA), Palm Beach, Aruba, Aug. 2014.

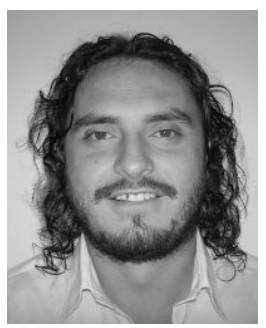

Dirk I.L. de Villiers (S'05-M'08) received the B.Eng and Ph.D. degrees in electrical and electronic engineering from the University of Stellenbosch, Stellenbosch, South Africa in 2004 and 2007 respectively.

From 2008 to 2009 he was a post-doctoral fellow at the University of Stellenbosch working on antenna feeds for the South African SKA program. He is currently a senior lecturer at the University of Stellenbosch, and has spent several months as research visitor at Antwerp University in Antwerp, Belgium, as well as at Chalmers University of Technology in Gothenburg, Sweden. Between 2010 and 2014 he has worked on contract for EMSS Antennas (Pty) Ltd in Stellenbosch, South Africa, on the design of the reflector optics for the MeerKAT and SKA radio telescopes. His main research interests include reflector antennas as well as the design of microwave components.

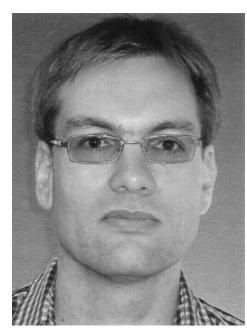

Robert Lehmensiek (S'98-M'01-SM'10) was born in Swakopmund, and received the BEng, MEng and $\mathrm{PhD}$ (Eng) in electronic engineering from the University of Stellenbosch, Stellenbosch, South Africa, in 1992, 1995, and 2001, respectively. From 1995 to 2002, he was a design engineer at Reutech Radar Systems, Stellenbosch, South Africa. In 2003, he joined EMSS Antennas, Stellenbosch, South Africa, where he has been mainly involved in the design and development of antennas. Since 2007, he has also taught antenna courses and supervised postgraduate students at the Cape Peninsula University of Technology, Bellville, South Africa. 